\title{
The environmental dependence of radio-loud AGN activity and star formation in the 2dFGRS
}

\author{
P. N. Best, ${ }^{1 \star}$ \\ ${ }^{1}$ Institute for Astronomy, Royal Observatory Edinburgh, Blackford Hill, Edinburgh EH9 3HJ, UK
}

2 August 2021

\begin{abstract}
By combining the 2-degree Field Galaxy Redshift Survey with the NRAO VLA Sky Survey at $1.4 \mathrm{GHz}$, the environments of radio loud AGN in the nearby Universe are investigated using both local projected galaxy densities and a friends-of-friends group finding algorithm. Radio-loud AGN are preferentially located in galaxy groups and poor-to-moderate richness galaxy clusters. The AGN fraction appears to depend more strongly on the large-scale environment (group, cluster, etc) in which a galaxy is located than on its more local environment, except at the lowest galaxy surface densities where practically no radio-loud AGN are found. The ratio of absorption-line to emission-line AGN changes dramatically with environment, with essentially all radio-loud AGN in rich environments showing no emission lines. This result could be connected with the lack of cool gas in cluster galaxies, and may have important consequences for analyses of optically-selected AGN, which are invariably selected on emission line properties. The local galaxy surface density of the absorption-line AGN is strongly correlated with radio luminosity, implying that the radio luminosities may be significantly boosted in dense environments due to confinement by the hot intracluster gas.

The environments of a radio-selected sample of star forming galaxies are also investigated to provide an independent test of optical studies. In line with those studies, the fraction of star forming galaxies is found to decrease strongly with increasing local galaxy surface density; this correlation extends across the whole range of galaxy surface densities, with no evidence for the density threshold found in some optical studies.
\end{abstract}

Key words: galaxies: active — galaxies: starburst — galaxies: evolution — radio continuum: galaxies

\section{INTRODUCTION}

The advent of large galaxy redshift surveys, especially the 2-degree Field Galaxy Redshift Survey (2dFGRS; Colless et al. 2001) and the Sloan Digital Sky Survey (SDSS; York et al. 2000; Stoughton et al. 2002) have revolutionised our understanding of the effect of local environment upon the evolution of galaxies. Understanding how galaxy properties, such as luminosities, morphologies, star formation rates and nuclear activity, depend upon the environment that a galaxy inhabits can place important constraints on models of galaxy formation and evolution, and allow the intrinsic properties of the galaxies to be separated from those that have been externally induced ('nature vs nurture').

It has been known for many years that star formation rates are strongly suppressed in the central regions of galaxy clusters (e.g. Dressler et al 1985), even if account is taken of the different distribution of morphological types in cluster environments as compared with the field. The large redshift surveys have shown that

^ Email: pnb@roe.ac.uk this suppression of the star formation rate is not only restricted to the extreme cluster environments, but begins at much lower environmental densities. Hashimoto et al. (1998) showed that the mean star formation rate shows a continuous correlation with local galaxy density, both inside and outside of clusters, using the Las Campanas Redshift Survey. Lewis et al (2002; hereafter Lew02) studied the fields around 17 clusters within the 2dFGRS, and found that the mean star formation rate of galaxies is relatively constant for projected galaxy surface densities below 1 galaxy per square $\mathrm{Mpc}$, but that at higher surface densities star formation is increasingly suppressed, down to essentially zero at 50 galaxies per square Mpc. A similar study using SDSS data broadly supports these conclusions although with a weaker break (Gómez et al. 2003; hereafter Gom03), and a study of SDSS field galaxies (Mateus \& Sódre 2003; hereafter MS03) is also in agreement at high surface densities, but suggests that the star formation rate remains sensitive to the local galaxy density even in more rarefied environments.

Regardless of the precise dependence at lower surface densities, environmental effects clearly function down to at least 1 galaxy per square Mpc, which is below both the mean galaxy surface den- 
sity at the virial radius of relatively rich clusters and that of galaxy groups. Therefore, the physical processes that lead to this quenching of star formation are not intrinsic to cluster environments (e.g. ram-pressure stripping of the interstellar medium by the hot cluster gas; Gunn \& Gott 1972), but also occur in smaller structures (cf. Martínez et al. 2002, who find that star formation is diminished even within galaxy groups of mass $M \sim 10^{13}$ in the $2 \mathrm{dFGRS)}$ ). The combination of this environmental dependence and the build up of galaxies into groups and clusters with cosmic time may be one of the drivers behind the decline in the mean cosmic star formation rate since redshifts $z \sim 1$ (Madau et al. 1998).

An alternative handle on galaxy activity and environmental influence comes through studies of active galactic nuclei (AGN). It is now apparent that essentially all massive galaxies in the nearby Universe host a supermassive black hole at their centres, whose mass is roughly proportional to the spheroidal mass of the galaxy (e.g. see review by Kormendy \& Gebhardt 2001). This suggests that the build-up of the central black hole and that of its host galaxy are fundamentally linked; the similarity of the cosmic evolution of the mean global star formation rate to that of the rate at which gas is accreted onto black holes (as estimated from the radio luminosity function; Dunlop 1998), at least out to redshifts $z \sim 1-2$ where both are well-determined, provides further evidence for this. Investigating how the incidence of AGN activity depends upon local environment can provide valuable insight into the origin of this connection, and also into the physical processes that trigger AGN activity.

To produce a powerful AGN the necessary ingredients are a massive black hole, a supply of fuel, and a transport mechanism to connect the two. If AGN activity is driven predominantly by the availability of the cold gas (the same factor that drives star formation activity) then AGN activity, like star formation, should be greatly suppressed in cluster environments. If instead the probability of AGN activity is solely a function of the central black hole, independent of the local environment of the galaxy, the AGN fraction would simply trace the distribution of galaxy bulges. Alternatively, at least for the most powerful AGN it has frequently been proposed that galaxy interactions or mergers may trigger the AGN activity (e.g. Hutchings \& Campbell 1983, Heckman et al. 1984, 1986), providing both a supply of gas and a mechanism for moving it to the central regions of the galaxy. In this case the dependence of AGN activity on environment on group- or cluster-scales would be less clear-cut, with AGN favouring those environments in which conditions are optimal for galaxy interactions and mergers.

Studies of the environmental dependence of AGN activity have a long and chequered history. Dressler et al. (1985) argued that AGN activity was suppressed in clusters, finding that only $1 \%$ of cluster galaxies showed AGN activity compared to $5 \%$ of field galaxies in their sample. ${ }^{\dagger}$ However, the redshift range of their cluster and field samples were very different, and the field sample contained many higher redshift AGN which were only bright enough to make it into their sample because of the magnitude boosting effect of the AGN itself. Biviano et al. (1997) find that if a correction is applied for this magnitude bias then the lower incidence of AGN activity in clusters is consistent with being due solely to the difference in the morphological mix between cluster and field. Carter

$\dagger$ Note that the fraction of galaxies which host AGN is very dependent upon both the depth of the observations and the details of the spectral definition of an AGN: Ho et al. (1997) classify as many as $43 \%$ of the galaxies in their survey of nearby bright $\left(B_{T}<12.5\right)$ galaxies as active. et al. (2001) similarly find little evidence for an environmental dependence of AGN fraction.

Using the 2dFGRS and the SDSS it is possible to go beyond a binary comparison of cluster against field and investigate the environmental dependence of AGN activity in much greater detail, as has been done for star formation activity. Kauffmann et al (2003) robustly selected a sample of AGN from the SDSS survey, by modelling the underlying stellar continuum to obtain accurate measurements of the Balmer emission lines, and then using emission line ratio diagnostics to separate the AGN from star forming galaxies. Kauffmann et al (in prep; private communication) find that these AGN, especially those with strong emission lines, are less common in regions of high galaxy surface density. However, Miller et al (2003), also using the SDSS, found that the fraction of galaxies hosting AGN remains roughly constant across all environments from cluster cores to the rarefied field.

The contradiction between these two sets of results indicates that selection of active galaxies by optical emission lines is neither straightforward nor uncontroversial. In addition, recent Chandra Xray observations of galaxy clusters have identified a population of $\mathrm{X}$-ray active cluster galaxies which would not be selected as active galaxies on the basis of either their optical or emission line properties (e.g. Martini et al. 2002), and radio studies (Miller \& Owen 2002) have similarly found a population of dust-obscured active and star-forming galaxies towards the central regions of clusters. Therefore, selecting active galaxies by means other than their emission line properties may provide more robust results, and will certainly provide a valuable test of the results currently being derived from the SDSS. An efficient way to do this is to use radio-loud AGN: these are straightforward to locate and study, and large-area deep radio surveys are already available. It must be borne in mind, however, that only 10-20\% of AGN are radio loud (e.g. Hooper et al. 1995), and these may represent a biased subset of the AGN population as a whole.

The environments of powerful radio sources have been widely studied at low redshifts (e.g. Prestage \& Peacock 1988; Hill \& Lilly 1991; Miller et al. 2002) and appear to favour galaxy groups and weak clusters; they tend to avoid the densest environments except in the few cases where they are in the special location of being hosted by the central dominant galaxy of a cluster. To date, though, these studies have been both limited to the most powerful radio sources and, generally, based upon galaxy number count statistics or cross-correlation analyses, with little or no spectroscopic redshift information (the Miller et al. study is the exception to this, providing redshifts for a handful of galaxies in the $\sim 20$ arcmin radius region around each of 25 low redshift radio sources). By comparing the 2dFGRS with a deep large-area radio survey such as the NRAO VLA Sky Survey (NVSS; Condon et al. 1998) it is possible to overcome both of these deficiencies, studying the spectroscopicallydetermined large-scale environments of 'typical' radio-loud AGN within the $2 \mathrm{dFGRS}$, and comparing these to those of the galaxy population in general. This is the goal of the current paper.

In Section 2, the galaxy and radio source samples used in the analysis are defined. The methods used to derive the properties of these galaxies are described in Section 3. The results of the environmental analysis are described in Section 4, and these are discussed in Section 5. Section 6 summarises the results. Throughout the paper, the values adopted for the cosmological parameters are $\Omega_{m}=0.3, \Omega_{\Lambda}=0.7$, and $H_{0}=65 \mathrm{~km} \mathrm{~s}^{-1} \mathrm{Mpc}^{-1}$. 


\section{GALAXY SAMPLES AND PROPERTIES}

\subsection{2dFGRS galaxy sample}

The primary galaxy sample for this study is drawn from the $2 \mathrm{dF}$ Galaxy Redshift Survey, described by Colless et al. (2001). The 2dFGRS obtained spectra through $\sim 2.1$ arcsec diameter fibres for nearly a quarter of a million galaxies brighter than a nominal extinction-corrected magnitude limit of $b_{J}=19.45$, in two declination strips (one equatorial and the other close to the south galactic pole; these are hereafter referred to as the NGP and SGP strips respectively) and a number of random 2-degree diameter fields. For this paper, analysis was restricted to those galaxies with reliable redshifts (quality $\geq 3$; cf. Colless et al. 2001) which lie within the NGP or SGP strip.

A cut in redshift was made to select those galaxies which lay in the redshift range $0.02<z<0.1$. At redshifts below $z \sim 0.02$ the physical size of the 2dFGRS survey region is too small to investigate the large-scale galaxy environments, whilst the upper redshift cut was set by the depth of the 2dFGRS survey, as discussed below. The absolute B-band magnitudes of these galaxies were calculated, using the average $\mathrm{K}$-correction for $2 \mathrm{dFGRS}$ galaxies derived by Madgwick et al. (2002); $K_{B}(z) \approx 1.9 z+2.7 z^{2}$. Only those galaxies with absolute magnitudes $M_{B}<-19$ (that is, $L_{B} \gtrsim 0.25 L_{B}^{*}$; cf Norberg et al 2002) were retained for subsequent analysis; this absolute magnitude limit corresponds roughly to the apparent magnitude limit of the survey at a redshift $z \sim 0.1$. Thus, the combination of absolute magnitude and redshift cuts largely removes any redshift biases from the sample.

This produced a sample of 56143 galaxies. However, not all of these galaxies are appropriate for environmental analysis, for example because they lie close to the boundaries of the survey, or in regions of low spectroscopic completeness. This was assessed for each galaxy individually during the process of local environment estimation through the 10th nearest neighbour method, as discussed in Section 3.1: about $10 \%$ of the galaxies were rejected from the sample during this analysis, due to their locations. Note that removal of these galaxies will not bias any of the results of the paper, firstly because these are largely a random subset of galaxies which happen to lie near the boundaries of the survey, and secondly because in any case these same cuts are applied to all of the samples under study.

By this process, a basis catalogue of 50684 galaxies with redshifts $0.02 \leq z \leq 0.10$ and absolute magnitudes $M_{B}<-19$ was derived, for each of which good estimates of the local galaxy density can be made.

\subsection{Radio source sample}

From this basis catalogue, subsamples of radio galaxies are constructed using the radio source sample of Sadler et al. (2002; hereafter Sad02). These authors cross-correlated an earlier version of the 2dFGRS, which contained almost $30 \%$ of the final 2dFGRS catalogue, with the radio sources in the NRAO VLA Sky Survey (Condon et al. 1998), which is a $1.4 \mathrm{GHz}$ survey to a limiting flux density of about $2.5 \mathrm{mJy}$ covering the entirety of the sky north of -40 degrees declination. Sad02 identified all of the single component radio sources which lay within 15 arcseconds of a galaxy with a redshift in the 2dFGRS catalogue, and also added to this sample a small number of double or multiple component radio sources for which an examination of a radio-optical overlay indicated a host galaxy within the 2dFGRS catalogue. For each of the 912 candidate radio source matches, they examined the optical emission and absorption line spectrum of the host galaxy, to classify the source as:

(i) 'star-forming' galaxies $(\mathrm{SF})^{\ddagger}$, where the spectrum is dominated by strong narrow Balmer emission lines.

(ii) AGN, separated into three subclasses: absorption-line ellipticals galaxies (Aa), absorption line galaxies with weak LINER-like emission lines (Aae), and galaxies with spectra dominated by nebular emission lines such as [OII] or [OIII] (Ae).

(iii) sources whose classifications are likely (SF?, Aa?, Ae?, Aae?) or completely uncertain (???).

These classifications were made by visual examination of the spectra, and their reliability was checked against Principle Component Analyses and emission line diagnostic diagrams (Sad02). ${ }^{\S}$

For the current analysis, stricter criteria need to be applied to these radio sources, in order to prevent false identifications from unduly influencing the results. Firstly, following the procedure of Sad02, the radio source sample is limited to only those radio sources with radio-optical offsets below 10 arcseconds, or in the case of resolved or multi-component radio sources with larger radio-optical offsets, only where the visual examination shows the identification to be secure. This restricts the size of the overall sample to 757 radio sources, of which up to $10 \%$ may be chance coincidences (Sad02). The radio source sample is further restricted to include only those galaxies in the final 2dFGRS sample constructed above, namely by restricting it to the redshift range $0.02 \leq$ $z \leq 0.10$, to galaxies with absolute magnitudes $M_{B}<-19$, and to galaxies for which local environments were able to be calculated, as discussed above. A final restriction was made to include only radio sources with radio luminosities $\uparrow$ above $10^{21} \mathrm{~W} \mathrm{~Hz}^{-1}$ at $1.4 \mathrm{GHz}$ rest-frame. This provided a final sample of 272 radio sources with optical counterparts. Of these, the proportion of chance coincidences is $\lesssim 5 \%$. This is reduced from the $10 \%$ in the original sample because of the various cuts applied to the data, especially the restriction to the most luminous optical galaxies; this is because the probability of an optical galaxy being a powerful radio source is an increasing function of optical luminosity (e.g. Sadler et al. 1989), and so the removal of the lowest optical luminosity galaxies removes a significant fraction of the chance alignments but very few of the genuine sources.

It is important that the star forming and AGN radio source subsamples are cleanly selected from these 272 radio sources. Naively assuming that all sources classified by $\mathrm{Sad02}$ as 'SF?' can be adopted as star forming galaxies, and all 'Ae?', 'Aa?' and 'Aae?' as AGN, may lead to some mis-classification which could strongly bias the results. The radio luminosity was therefore used to provide a second handle on the source classification. The majority of low

$\ddagger$ Star forming galaxies emit at radio wavelengths, predominantly due to the synchrotron emission of particles accelerated in supernova shocks. The radio luminosity is roughly correlated with the star formation rate: a 1.4 GHz radio luminosity of $10^{22} \mathrm{~W} \mathrm{~Hz}^{-1}$ corresponds to a star formation rate of order $5 M_{\odot} \mathrm{yr}^{-1}$ (e.g. Condon 1992 and references therein; Carilli 2001).

$\S$ Note that because the $2 \mathrm{dFGRS}$ spectra are uncalibrated, it is not possible to classify the galaxies in a more robust way, such as decomposing the stellar continuum to investigate the presence of a young stellar population, or modelling and subtracting the stellar continuum to prevent stellar absorption lines from affecting the emission line properties (cf. Kauffmann et al. 2003).

ๆ A radio spectral index of $\alpha=0.8$ (for $S_{\nu} \propto \nu^{-\alpha}$ ) was adopted for the $\mathrm{K}$-corrections in calculating the rest-frame radio luminosities. 
luminosity radio sources are star forming while at high luminosities AGN dominate (e.g. Sad02; Miller \& Owen 2002; Machalski \& Godlowski 2000). The transition between the two classes is gradual and the exact location at which it occurs is still a matter of debate with Sad02 favouring $L_{1.4 \mathrm{GHz}} \approx 10^{23} \mathrm{~W} \mathrm{~Hz}^{-1}$ and Miller \& Owen favouring the lower value of $10^{22.7} \mathrm{~W} \mathrm{~Hz}^{-1}$. This difference may arise because the Miller \& Owen study concentrates upon cluster environments and, as discussed in the introduction, star formation activity is known to be suppressed there, decreasing the proportion of star forming galaxies.

A luminosity of $L_{1.4 \mathrm{GHz}} \approx 10^{22.8} \mathrm{~W} \mathrm{~Hz}^{-1}$ is here adopted for the transition point. The subsample of star forming galaxies is taken to include all of these galaxies securely classified by Sad02 as star forming galaxies ('SF'), plus those galaxies classified as likely star forming galaxies ('SF?') which also have a radio luminosity below this transition value (that is, likely star forming galaxies based upon their emission lines, and also more likely to be star forming based upon their radio luminosities). 154 radio sources satisfy these criteria, with $1.4 \mathrm{GHz}$ radio luminosities ranging from $3 \times 10^{21}$ to $4 \times 10^{23} \mathrm{~W} \mathrm{~Hz}^{-1}$. The AGN subsample is taken to include those sources securely classified as AGN by Sad02 (classes Aa, Ae, and Aae), together with those sources classified as likely AGN (Aa?, Ae?, Aae?) with radio luminosities above the cut-off; there are 91 sources thus classified as AGN, with radio luminosities ranging from $10^{22}$ to $1.4 \times 10^{25} \mathrm{~W} \mathrm{~Hz}^{-1}$. This additional radio luminosity selection may not avoid all misclassifications, but does greatly reduce the likelihood of them. It naturally leads to the exclusion of a small subset of perfectly good radio sources ('SF?' sources with $1.4 \mathrm{GHz}$ radio luminosities brighter than $10^{22.8} \mathrm{~W} \mathrm{~Hz}^{-1}$, and 'Aa?', 'Ae?' and 'Aae?' sources fainter than that luminosity) but their exclusion is unlikely to bias the results significantly, and it is considered preferable to work with a smaller but cleaner sample than to risk including mis-classified objects.

It is finally important to note that the classification method of Sad02 takes no account of the recent results of Kauffmann et al. (2003), that many AGN are found to be composite systems which also contain star formation activity. Since a fibre instrument such as the $2 \mathrm{dF}$ samples a large fraction of the galaxy light, the star forming component is likely to dominate the emission line properties of such composite objects, and they are more likely to be classified as star forming galaxies. There may therefore be some composite $\mathrm{SF}+\mathrm{AGN}$ systems found within the SF class: however, the relatively lower radio luminosities of the SF galaxies (together with the substantially different environmental properties found in this paper) suggest that such objects are relatively rare. It is plausible that many of the 27 objects rejected as having uncertain classifications are composite objects.

Two further samples of radio sources were also defined: (i) Luminous AGN, defined to be the subset of the AGN class which also had $L_{1.4 \mathrm{GHz}} \geq 10^{23} \mathrm{~W} \mathrm{~Hz}^{-1}$, and (ii) Extended radio sources, defined as the subset of AGN for which the NVSS radio emission was extended. These two subsamples contain 40 and 25 radio sources respectively. 21 of the 25 extended radio sources also satisfy the luminous AGN definition, and so the extended radio sources are largely, but not entirely, a subset of the luminous AGN class.

\subsection{Parent galaxy sample}

As discussed above, the Sad02 radio sample was not constructed from the entirety of the 2dFGRS survey, but from only the first $\sim 30 \%$ observed. Ideally the radio source samples defined from the Sad02 regions should therefore be compared with the parent galaxy sample used by Sad02, and not with the entire 2dFGRS region, since otherwise there is the possibility of introducing biases if the initial regions studied by the $2 \mathrm{dFGRS}$ were not fully representative of the complete survey region. However, the parent catalogue of Sad02 is not available. Whether the Sad02 parent sample is biased with respect to the final survey catalogue can be investigated by comparing the final 2dFGRS galaxy catalogue with the subsample of galaxies that were included in the first catalogue release of the 2dFGRS (100,000 galaxies, of which the Sad02 sample comprised 70\%). Results of the environmental analyses discussed in Section 3 were compared for the final 2dFGRS galaxy sample and the first 100,000 galaxy subsample. One notable difference was found between the two samples: a significantly smaller percentage of the galaxies in the 100,000 galaxy catalogue are found the very highest local densities (richest clusters) than in the final release catalogue. Further investigation shows that this difference arises predominantly because of a single supercluster environment (at RA $13 \mathrm{~h}$, Dec $-2^{\circ}, z \sim 0.084$ ) which was very poorly covered in the original data release.

Apart from this one extreme environment, no systematic differences were found between the two samples in either local densities or group / cluster environments. Therefore, given that the Sad02 parent sample comprises $\sim 70 \%$ of the 100,000 galaxy release, and that the 100,000 galaxy release contains no especially extreme environments, any biases between the Sad02 parent sample and the first-release 100,000 galaxy sample are likely to be negligible. Therefore, the parent galaxy sample adopted for comparison with the radio source samples comprises the 21085 galaxies from the 50684-galaxy basis catalogue described above, which were present in the first 100,000 galaxy release of the 2dFGRS.

\subsection{Morphological sample}

The SDSS First Data Release (Abazajian et al. 2003) overlaps in part with the NGP strip of the 2dFGRS. For those galaxies in the overlap region, their morphological properties can be investigated using the SDSS 'concentration parameter', C. This parameter is defined as the ratio of the radius enclosing $90 \%$ of the galaxy light in the $r$-band to that containing $50 \%$ of the light (cf. Stoughton et al. 2002): most early-type galaxies have $C>2.6$ while spirals and irregulars typically have $2.0<C<2.6$ (Strateva et al. 2001).

For the 50684 galaxies in the final 2dFGRS sample, a search was made within the SDSS catalogue for any galaxy within 5 arcsec in positional offset $(\approx 10 \mathrm{kpc}$ at redshift 0.1$)$, and within $340 \mathrm{~km} \mathrm{~s}^{-1}$ in redshift (four-times the rms deviation of $85 \mathrm{~km} \mathrm{~s}^{-1}$ between SDSS and 2dFGRS measured redshifts; cf Norberg et al 2002). The completeness and reliability of this cross-correlation were estimated by varying the size of the acceptable offsets in both velocity and position, and comparing both the total number of 2dFGRS-SDSS matches and the number of incidences whereby two SDSS galaxies are consistent with the 2dFGRS galaxy; it is estimated that for allowable offsets of $5 \operatorname{arcsec}$ and $340 \mathrm{~km} \mathrm{~s}^{-1}$ the cross-correlation of the two surveys is about $98 \%$ complete and 99.9\% reliable.

For all galaxy matches, the concentration parameter $\mathrm{C}$ was calculated from the SDSS $r$-band data, and the galaxy was classified as either early or late-type. Although this was only possible for 5781 galaxies, this is sufficient to determine the morphological mix of 2dFGRS galaxies as a function of environment. 


\section{ENVIRONMENTAL PARAMETERS}

The local environment of each galaxy in the survey was investigated using two different methods, which are described below.

\subsection{0th-nearest neighbour estimate}

For each galaxy with redshift $0.02 \leq z \leq 0.10$ and absolute magnitude $M_{B}<-19$, the local projected galaxy density was calculated using an adaptation of the 10th-nearest neighbour approach which has been commonly used for this purpose (cf. Dressler et al. 1980, Lew02, Gom03, Miller et al. 2003). Specifically, for each galaxy, a redshift shell of $\pm 1000 \mathrm{~km} \mathrm{~s}^{-1}$ centred on the redshift of that galaxy is considered, and the projected distance $\left(r_{10}\right)$ to the 10th nearest neighbour [also having $M_{B}<-19$ ] within that redshift shell in the basis catalogue is calculated. This distance is then converted into a local projected galaxy density, $\Sigma=10 / \pi r_{10}^{2}$.

Several factors deserve deeper consideration in this determination of the surface densities. The first is the adoption of a fixed velocity range of $1000 \mathrm{~km} \mathrm{~s}^{-1}$ in the definition of the redshift shell, to reject foreground and background galaxies. This velocity range is well-suited to 'average' environments, but in the richest clusters the large velocity dispersions may lead this method to underestimate the local galaxy density, due to some companions falling outside of this range. However, this is unavoidable without making the velocity shell so wide as to include unacceptable numbers of foreground and background galaxies. Lew02 got around this problem by limiting their analysis to cluster environments, and using a variable-width velocity shell set to be three times the velocity dispersion of each cluster. However, this method does not allow analysis of field environments, limiting the range of surface densities that can be studied, and in addition it may introduce biases at the lowest surface densities (see discussion in Section 5.1). An alternative method for investigating the environments of field galaxies is to convert redshifts into distances and calculate the full threedimensional local density of the galaxies (e.g. see MS03); however, peculiar velocities make this method very poor in cluster environments. Overall the adoption of a fixed velocity range provides a method that works reliably across all environments, with sufficient accuracy for the science goals of this paper. The reliability of this technique was further tested by varying both the width of the velocity shell $\left( \pm 500,1000,1500 \mathrm{~km} \mathrm{~s}^{-1}\right)$ and the number of neighbours out to which the result was evaluated $(5,10,15)$ : although the projected surface densities of individual galaxies changed, the overall sample-average results were broadly unaffected.

A second effect concerns the boundaries of the 2dFGRS catalogue, and the variations in both redshift completeness and magnitude limit of the catalogue as a function of position. The redshift survey covers two long strips in declination, each of which is only about 10 degrees wide, meaning that all galaxies lie within 5 degrees of a catalogue boundary, and many lie significantly closer. To estimate the effect that this will have on the surface density calculation, the following procedure was followed:

(i) For each galaxy, the value of $r_{10}$ was calculated as above. 10000 positions were then randomly chosen within a circle of radius $r_{10}$ centred on that galaxy.

(ii) For each of these 10000 positions, the redshift completeness of the 2dFGRS catalogue was evaluated for galaxies brighter than the apparent magnitude corresponding to an absolute magnitude of $M_{B}=-19$ at the redshift of the galaxy under study. This calculation was carried out using the redshift completeness, limiting magnitude, and $\mu$-masks (see Colless et al. 2001 for details) provided by the 2dFGRS team. If a given position lay outside the 2dFGRS region of study, or within one of the survey holes associated with bright stars, then the redshift completeness was set to zero for that position.

(iii) An average redshift completeness was derived for these 10000 positions. In this way, for each galaxy the average redshift completeness for galaxies brighter than $M_{B}<-19$ over the region of sky out to radius $r_{10}$ was estimated.

(iv) Where the average redshift completeness was below $50 \%$, or the value of $r_{10}$ was in excess of 5 degrees, the galaxy was removed from further analysis; these are predominantly galaxies near the boundaries of the survey region. Removal of these galaxies will not bias any of the results of the paper, since these same cuts are applied to all of the samples under study.

(v) The surface densities calculated for the remainder of the galaxies were scaled up by the inverse of their average redshift completeness. This correction factor provides a rough first-order approximation to the correction and, since all applied corrections are below a factor of 2 , any inaccuracies will have a negligible effect on the final results.

\subsection{Group or cluster membership}

The 10th nearest neighbour analysis technique was developed at a time before large spectroscopic catalogues were available, and foreground and background field galaxies had to be statistically subtracted. The use of redshift information to remove clearly unrelated galaxies greatly improves the reliability of the analysis, and there are clearly many advantages over simple comparisons with, for example, cluster-centric radius. However, this technique still discards some of the information which is available in a complete spectroscopic dataset, especially in galaxy groups or clusters with over 10 members. Using the velocity information available in the $2 \mathrm{dF}$ GRS and SDSS catalogues, it is in principle possible to determine for each individual galaxy its exact environment: is it an isolated galaxy, a member of a small group, on the outskirts of a cluster, within a cluster core, etc?

Catalogues of galaxy groups have been constructed from the 2dFGRS by both Merchán \& Zandivarez (2002) and by Norberg et al. (2003), in both cases using an adapted version of the friendsof-friends approach first described by Huchra \& Geller (1982). The basis of this approach is as follows. Two galaxies with an angular separation on the sky of $\theta_{12}$, and recession velocities of $V_{1}$ and $V_{2}$ (with a mean, $V=\left(V_{1}+V_{2}\right) / 2$ ) are considered to be linked if they satisfy the following conditions:

$2 \sin \left(\frac{\theta_{12}}{2}\right) \frac{V}{H_{0}} \leq D_{\mathrm{L}}$,

$\left|V_{1}-V_{2}\right| \leq V_{\mathrm{L}}$

where $D_{\mathrm{L}}$ and $V_{\mathrm{L}}$ are the transverse and radial linking lengths respectively. For a survey of fixed apparent magnitude limit, in the standard Huchra \& Geller formalism the linking lengths $D_{\mathrm{L}}$ and $V_{\mathrm{L}}$ vary with recession velocity in order to compensate the change in the sampling of the galaxy luminosity function with distance, and thus to provide equal sensitivity to groups across all redshifts. The linking lengths scale as $D_{\mathrm{L}}=D_{0} R$ and $V_{\mathrm{L}}=V_{0} R$, where $D_{0}$ and $V_{0}$ are the linking lengths at some fiducial recession velocity $V_{\mathrm{f}}$, and $R$ is a scaling factor given by:

$R=\left[\frac{\int_{-\infty}^{M_{12}} \phi(M) \mathrm{d} M}{\int_{-\infty}^{M_{\lim }} \phi(M) \mathrm{d} M}\right]^{-1 / 3}$, 
where $M_{\text {lim }}$ and $M_{12}$ are the absolute magnitudes of the faintest galaxy visible at distances of $V_{\mathrm{f}} / H_{0}$ and $V / H_{0}$ respectively, and $\phi(M)$ is the galaxy luminosity function of the sample.

In the present analysis, such a scaling factor is in principle unnecessary because the adoption of a fixed absolute magnitude limit $\left(M_{B}<-19\right.$, as discussed above) removes this distance dependence of the luminosity function sampling (ie. $M_{\text {lim }}$ and $M_{12}$ are both equal to -19 , making $R$ unity). However, the 2dFGRS has a slightly varying magnitude limit, and the spectroscopic completeness varies with position on the sky. These do require (minor) correction (cf. Merchán \& Zandivarez 2002), and can be accounted for by redefining the scaling factor as

$R=\left[\frac{C_{1}+C_{2}}{2}\right]^{-1 / 3}$

where $C_{1}$ and $C_{2}$ are the redshift completeness for magnitudes brighter than the apparent magnitude corresponding to $M_{B}=-19$ for the redshift of the galaxies in question, at the location in the $2 \mathrm{dFGRS}$ of two galaxies whose linkage is being considered. These redshift completeness values can be evaluated using the redshift completeness, limiting magnitude, and $\mu$-masks provided by the 2dFGRS team (see Colless et al. 2001 for details).

The transverse linking length, $D_{\mathrm{L}}$, is set by defining groups to be those regions with a mean galaxy density contrast $\delta \rho / \rho$ in excess of 80 (cf. Merchán \& Zandivarez 2002; Ramella, Pisani \& Geller 1997). This relates to $D_{0}$ through the expression

$\frac{\delta \rho}{\rho}=\frac{3}{4 \pi D_{0}^{3}}\left(\int_{-\infty}^{M_{\lim }} \phi(M) \mathrm{d} M\right)^{-1}-1$.

Taking the Schechter fit to the luminosity function of 2dFGRS galaxies $\left(\phi^{*}=4.6 \times 10^{-3} \mathrm{Mpc}^{-3}, \alpha=-1.21, M_{B}^{*}=-20.59\right.$, for $H_{0}=65 \mathrm{~km} \mathrm{~s}^{-1} \mathrm{Mpc}^{-1}$; Norberg et al. 2002), for an absolute magnitude limit of $M_{B}=-19$, a fiducial transverse linking length of $D_{0}=1.25 \mathrm{Mpc}$ is derived.

Regarding the velocity linking length, $V_{\mathrm{L}}$, this needs to be larger than the equivalent distance linking length because peculiar velocities smear galaxies out along the distance axis. As discussed by Nolthenius \& White (1987), there is a problem if the standard Huchra \& Geller approach of scaling the velocity linking length with distance according to Equation 1 is applied to surveys sampling to moderate redshifts: if a fiducial velocity linking length is chosen which is large enough to cope with peculiar motions in nearby galaxies, then when this is scaled with distance it quickly becomes so large that distant groups are seriously contaminated by foreground and background galaxies. $\|$ The current analysis avoids this problem, since the adoption of a fixed absolute magnitude limit essentially removes the requirement of this scaling. All that is required here is to choose a suitable value of $V_{0}$.

Nolthenius \& White (1987) find that the balance between retaining valid group members and minimising contamination from

॥ For example, Merchán and Zandivarez (2002) determine a best-fit value of $V_{L}=200 \mathrm{~km} \mathrm{~s}^{-1}$ (at their fiducial recession velocity $V_{f}=$ $1000 \mathrm{~km} \mathrm{~s}^{-1}$ ) for their analysis of 2dFGRS groups, by using mock catalogues to investigate the reliability and completeness of different values. However, using the scaling of Equation 1, this corresponds to a linking length of about $900 \mathrm{~km} \mathrm{~s}^{-1}$ for the absolute magnitude limit of $M_{B}=-19$ used in this paper. This value is clearly too large, and would lead to the identification of many spurious groups. Indeed, since Merchán and Zandivarez do use this scaling, and study groups out to $z \sim 0.25$, their linking length is very large at higher redshifts and their group catalogue is likely to be highly contaminated beyond $z \sim 0.07$. non-members is optimised for a velocity linking length of between 1.5 and 2 times the typical velocity dispersion of groups. Merchán and Zandivarez (2002) and Norberg et al. (2003) have determined the average velocity dispersion of galaxy groups in the $2 \mathrm{dFGRS}$ to be 250 and $225 \mathrm{~km} \mathrm{~s}^{-1}$, respectively. Therefore, a fiducial velocity linking length of $V_{0}=450 \mathrm{~km} \mathrm{~s}^{-1}$ was adopted here.

In this way, the friends-of-friends mechanism was used to link pairs of galaxies and hence build up a catalogue of groups. $25 \%$ of the $M_{B}<-19$ galaxies are found to be isolated galaxies (lower than the $\sim 45 \%$ fraction that Norberg et al. derived for all 2dFGRS galaxies, because more massive galaxies are preferentially found in groups or clusters), $22 \%$ in 'groups' of 2 or 3 galaxies, a further $28 \%$ in groups of $4-15$ galaxies and $25 \%$ in still richer structures.

A robust estimate of the velocity dispersion of these galaxy groups was obtained using the 'gapper' estimator (Wainer \& Thissen 1976) for groups with less than 15 members and the 'biweight' estimator for larger groups (cf. Beers, Flynn \& Gebhardt 1990; Girardi et al. 1993). The virial radius and the mass of the group are then calculated according to the method of Giraldi \& Giuricin (2000). The median velocity dispersion of groups with 4 or more members is $220 \mathrm{~km} \mathrm{~s}^{-1}$, with a mean radius of $2.3 \mathrm{Mpc}$ and a mean mass of $5 \times 10^{13} M_{\odot}$. All of these values are consistent with previous determinations of the properties of nearby groups.

\section{RESULTS}

\subsection{Galaxy Surface Densities}

Figure 1 shows the variation with local projected galaxy surface density of the fraction of galaxies which host radio sources associated with AGN activity, and the equivalent fraction which host starforming radio sources. The environmental dependence of these two samples is remarkably different.

The star-forming galaxies show a strong correlation with environment in the sense that star formation is suppressed in high density environments; this is the result which is well-known at optical wavelengths, but which is now independently confirmed using a radio-based study. There is no evidence for a break in the correlation below $\sim 1 \mathrm{Mpc}^{-2}$, as was found by Lew02; the dependence upon environment continues to the lowest surface densities. These results are interpreted in Section 5.1.

The radio-loud AGN fraction shows very little dependence upon environment, with only the lowest density bin deviating significantly from a flat distribution. The surface density bins were defined to contain roughly equal numbers of AGN, in order to optimize the signal-to-noise; this also minimises the scatter between the data points a little bit, possibly giving a overly flat appearance. To test this, a Kolmogorov-Smirnov (KS) two-population test was applied to the surface density distribution of the AGN as compared to that of all galaxies. The probability that the AGN are not simply drawn randomly from all galaxies is $97 \%$ (ie. a $\sim 2.5 \sigma$ result). This result is largely driven by the lack of AGN at the lowest surface densities: the lowest surface density of galaxies around an AGN is $0.08 \mathrm{Mpc}^{-2}$ whilst over $8 \%$ of the $2 \mathrm{dFGRS}$ galaxy population have local galaxy densities below this value. Considering only galaxies with galaxy surface densities above $0.1 \mathrm{Mpc}^{-2}$, the significance of any difference between the AGN and all galaxies is below $1 \sigma$.

This apparent lack of any dependence of AGN activity on galaxy surface density hides a considerable quantity of information on AGN activity that can be uncovered in more detailed analysis. In Figure 2 this dependence of AGN fraction is shown again, and 


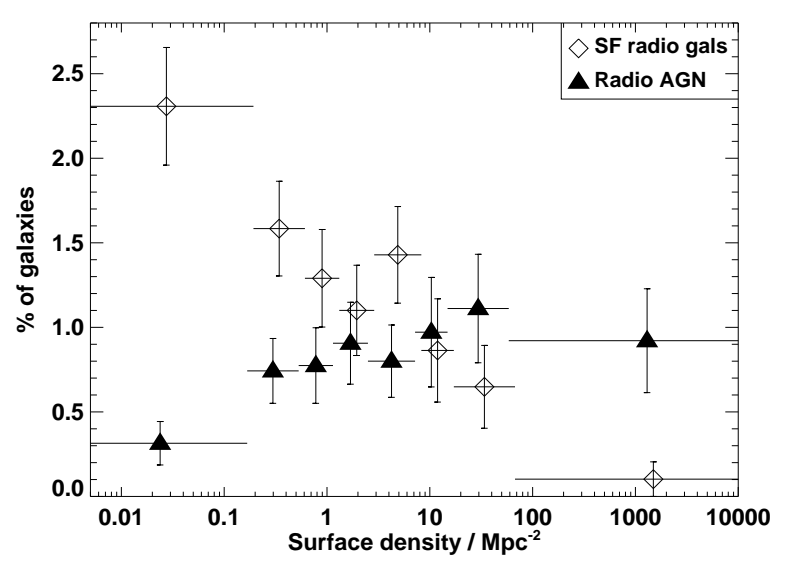

Figure 1. The fraction of galaxies within the 2dFGRS catalogue that are associated with radio sources securely classified as either star forming or AGN, as a function of the local projected galaxy surface density. The error bars plotted (here and in subsequent figures) correspond to the simple Poissonian uncertainty. The frequency of star forming radio sources is greatly suppressed in dense environments, whilst AGN activity is roughly independent of environment, except possibly in the most rarefied regions. [Note: in this and some subsequent figures, data points of different samples are offset an equal and opposite small distance along the $\mathrm{x}$-axis for clarity].

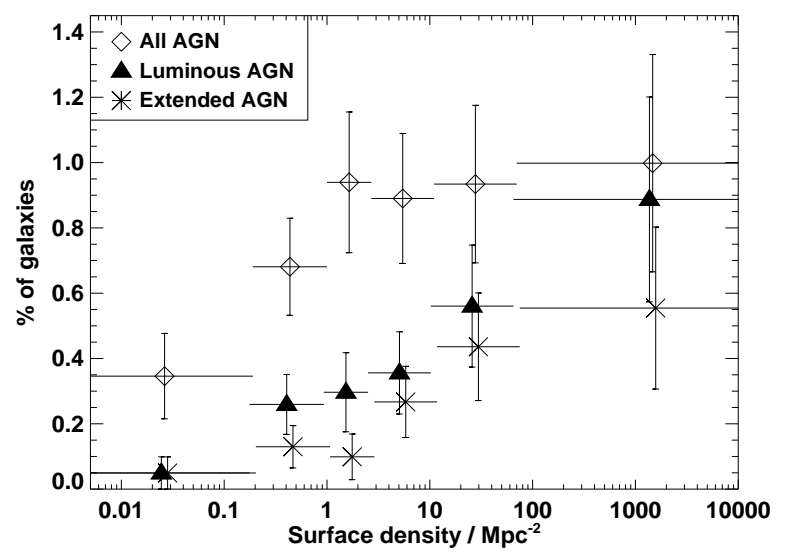

Figure 2. The fraction of galaxies within the 2dFGRS catalogue that are associated with radio-loud AGN, as a function of the local projected galaxy surface density, compared to the equivalent fractions considering only luminous AGN (those with $1.4 \mathrm{GHz}$ radio luminosities above $10^{23} \mathrm{~W} \mathrm{~Hz}^{-1}$ ) or only those AGN whose radio emission is extended in the NVSS data. Both luminous AGN and extended AGN are significantly more common in richer environments.

compared to the equivalent fraction for the two subsets of 'Luminous AGN' and 'Extended AGN', described in Section 2.2. The fraction of galaxies hosting AGN associated with each of these two classes shows a marked increase with increasing local galaxy density; KS tests show them to be inconsistent with flat distributions at the 99.5 and $99.7 \%$ significance levels respectively.

This correlation between AGN luminosity and local environment is perhaps better illustrated in Figure 3 which shows the projected surface density versus the radio luminosity for all of the AGN in the sample, separated into the three AGN classes defined by Sad02. Overall, a Spearmann-Rank correlation test indicates that radio luminosity and surface density are correlated at

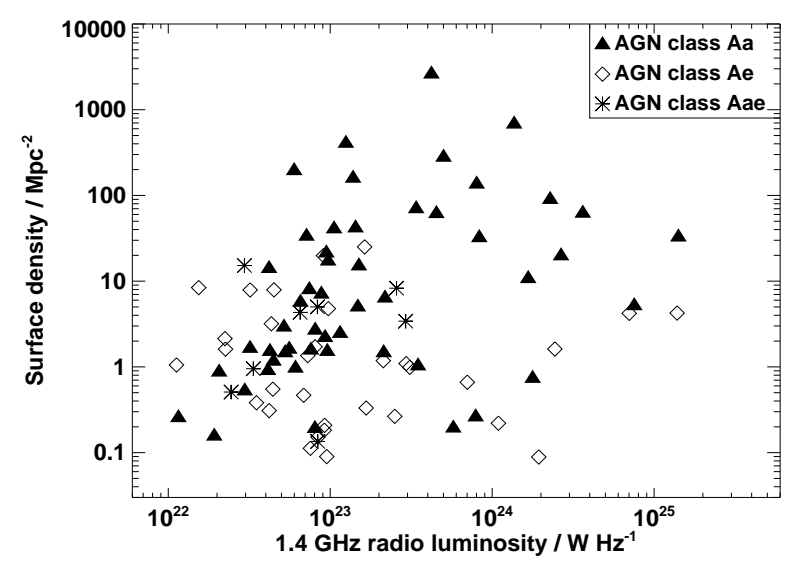

Figure 3. The local projected galaxy surface density of AGN plotted against their $1.4 \mathrm{GHz}$ radio luminosity, with the three different symbols showing the three different AGN classes separately. The absorption line AGN (Aa class) have surface densities strongly correlated ( $>99.9 \%$ significance) with radio luminosity, while the two emission line AGN classes (Ae and Aae) show no such correlation. Indeed, emission line AGN of all radio luminosities avoid the richest environments. The Aae radio galaxies all have relatively low radio luminosities.

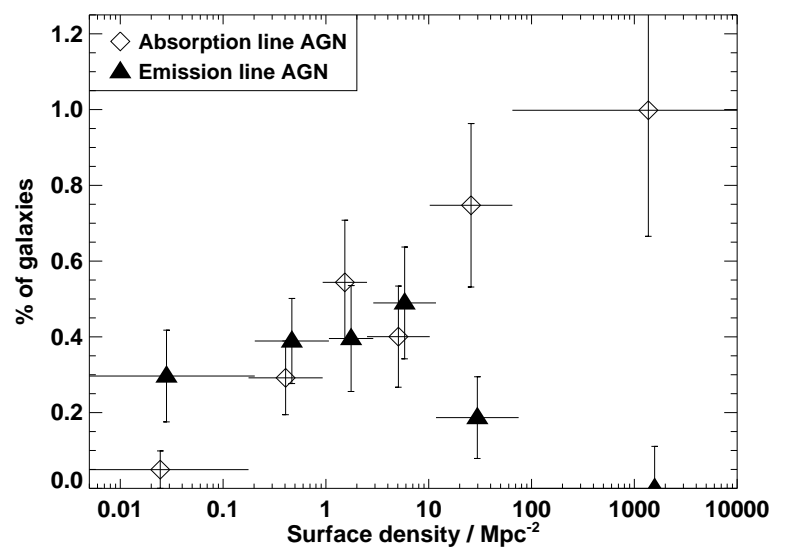

Figure 4. The fraction of galaxies within the 2dFGRS catalogue that are associated with radio-loud AGN as a function of the local projected galaxy surface density, separated into emission-line AGN (Ae and Aae classes of Sad02) and absorption line AGN (Aa class). The absorption line AGN are preferentially located in richer environments, whilst the emission line AGN avoid dense regions.

the $99.6 \%$ significance level. However, the most striking aspect of this plot is the difference between the properties of the three classes of AGN. The absorption-line AGN (class Aa) show a strong correlation between radio luminosity and galaxy surface density ( $>99.95 \%$ significance, using a Spearmann-Rank correlation test), but the emission-line AGN (class Ae) have no such correlation: these AGN cover the full range of radio luminosities, but completely avoid rich environments, showing the same range of low galaxy surface densities at all radio luminosities. The Aae class of AGN, which show both absorption features and weak emission lines, are relatively rare. These occupy the region of the diagram where the Aa and Ae classes overlap, namely, they are confined to regions of relatively low radio luminosity and low galaxy den- 
sity. The restriction of the Aae class to low radio luminosities may be because at higher radio luminosities their emission lines would have been stronger (due to the radio luminosity versus emission line luminosity correlation for emission line radio sources; cf Rawlings \& Saunders 1991), and if these dominated the spectrum the AGN would have been classified as Ae AGN. Given that the Aae AGN have surface densities comparable to the Ae class, in the analyses that follow these two classes are often considered together as emission-line AGN.

The percentage of galaxies in each of the absorption and emission line AGN classes, as a function of galaxy surface density, is further illustrated in Figure 4: while absorption-line AGN prefer richer environments, emission line AGN avoid them. The surface density distributions of the two samples differ at the $99.8 \%$ significance level. It is tempting to ascribe this difference to differences in the host galaxies of the AGN: the absorption-line AGN must all be hosted by elliptical galaxies, and such galaxies are common in cluster environments, but emission-line AGN could plausibly have a range of host galaxy types. However, as Figure 5 shows, there are no significant differences between the emission and absorptionline AGN in terms of their radio and optical luminosities, suggesting that there are no fundamental differences in host galaxies. Table 1 provides mean values for various properties of these AGN: there is only a 0.24 magnitude $(\lesssim 2 \sigma)$ difference in mean host galaxy absolute magnitude between the Aa and Ae classes, whilst the difference in mean local galaxy surface density is at the $\gtrsim 5 \sigma$ level. Possible explanations for the differences between the emission and absorption line AGN, and the consequences of these results, are discussed in Section 5.3.

Comparing the properties of the AGN with those of the elliptical galaxy sample selected from the SDSS data (Table 1), the mean local surface densities are similar. Figure 6 shows the fraction of all elliptical galaxies (statistically constructed from the morphological mix as a function of environment derived from the SDSS subsample) that host absorption-line AGN as a function of galaxy surface density. Because of the prevalence of early-type galaxies in clusters this distribution is flattened from the equivalent fraction in all galaxies, but it is still evident that absorption-line AGN have a weak preference for richer environments, even compared to elliptical galaxies in general.

\subsection{Group and Cluster membership}

The fractions of galaxies that host radio-loud AGN activity or radio-selected star formation activity are shown in Figure 7 as a function of the size of group or cluster in which that galaxy lies (as determined by the friends-of-friends analysis; see Section 3.2). Star forming galaxies prefer to avoid richer environments, although this dependence is clearly weaker than that on local environment found above. Star forming galaxies are also relatively rare in isolated galaxies: this latter result is most likely related to the radio selection, which picks out only galaxies with relatively high star formation rates ( $\left.\gtrsim 5 M_{\odot} / \mathrm{yr}\right)$. AGN are most common in moderate groups and poor clusters. The fraction of galaxies with AGN activity is lower in relatively isolated environments (1-3 galaxies) and also in the richest clusters ( $>50$ galaxies); a KS test gives a probability of $99.2 \%$ that the AGN are not simply randomly drawn from all galaxies.

By sub-classifying the AGN into emission-line AGN and absorption-line AGN (cf. Figure 8) it is apparent that the falloff in the AGN fraction in rich environments happens earlier for emission-line AGN than absorption-line AGN. The former are es-

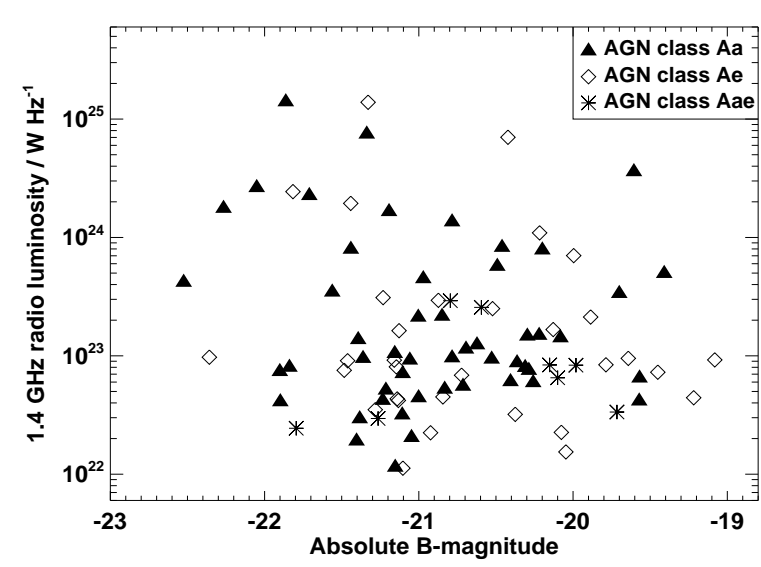

Figure 5. The B-band absolute magnitude of the AGN plotted against their $1.4 \mathrm{GHz}$ radio luminosity, with the three different symbols showing the three different AGN classes separately. Apart from the Aae class being confined to low radio luminosities, there are no statistically significant differences between the host galaxies of the three classes.

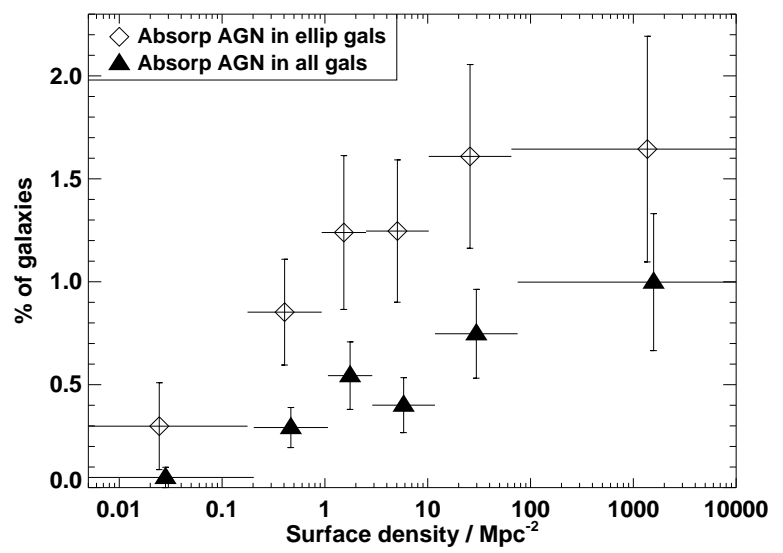

Figure 6. The fraction of radio-loud absorption-line AGN amongst all galaxies within the $2 \mathrm{dFGRS}$ catalogue, as a function of the local projected galaxy surface density, compared against the equivalent fraction amongst only early-type galaxies (since all the absorption line AGN will be hosted by early-type galaxies). The prevalence of early-type galaxies in rich environments means that, although with increasing local galaxy density an increasing fraction of galaxies host absorption-line AGN, early-type galaxies host absorption-line AGN with comparable probabilities across all environments except in the most rarefied regions.

sentially absent from all clusters of more than 20 galaxies, whilst the latter still strongly populate the moderate clusters (21-50 galaxies) and are only absent from the very richest clusters. A KS two-sample test indicates that, overall, the difference between the group/cluster environments of the emission and absorption-line AGN is significant at the $90 \%$ level, but this difference is concentrated in this single 'moderate cluster' environment, in which the contrast is much more significant (an emission to absorption-line AGN ratio of 1:10, compared to approximately $1: 1$ in poorer environments).

The low fraction of galaxies associated with AGN in relatively isolated environments is in agreement with the results of the local galaxy surface density analysis, in which a drop in the AGN frac- 
Table 1. The number of objects in various galaxy and AGN samples, together with the mean values of various properties of these galaxies.

\begin{tabular}{lccccc}
\hline Radio source type & $\mathrm{N}$ & $\mathrm{z}$ & $\begin{array}{c}\log _{10} L_{1.4 \mathrm{GHz}} \\
\mathrm{W} \mathrm{Hz}^{-1}\end{array}$ & $M_{B}$ & $\begin{array}{c}\log _{10}\left(\mathrm{Surf}^{\mathrm{Dens}}\right) \\
\mathrm{Mpc}^{-2}\end{array}$ \\
\hline AGN Class Aa & 51 & 0.073 & $23.25 \pm 0.10$ & $-20.91 \pm 0.10$ & $0.87 \pm 0.14$ \\
AGN Class Ae & 32 & 0.069 & $23.14 \pm 0.13$ & $-20.67 \pm 0.14$ & $0.01 \pm 0.12$ \\
AGN Class Aae & 8 & 0.071 & $22.86 \pm 0.14$ & $-20.55 \pm 0.25$ & $0.34 \pm 0.24$ \\
\hline SF galaxies & 154 & 0.051 & $22.45 \pm 0.03$ & $-20.48 \pm 0.06$ & $-0.17 \pm 0.07$ \\
\hline All galaxies & 50684 & 0.076 & - & $-19.99 \pm 0.01$ & $0.35 \pm 0.01$ \\
All gals (100k sample) & 21085 & 0.075 & - & $-20.00 \pm 0.01$ & $0.26 \pm 0.02$ \\
Early-types & 3746 & 0.078 & - & $-20.23 \pm 0.01$ & $0.82 \pm 0.02$ \\
\hline
\end{tabular}

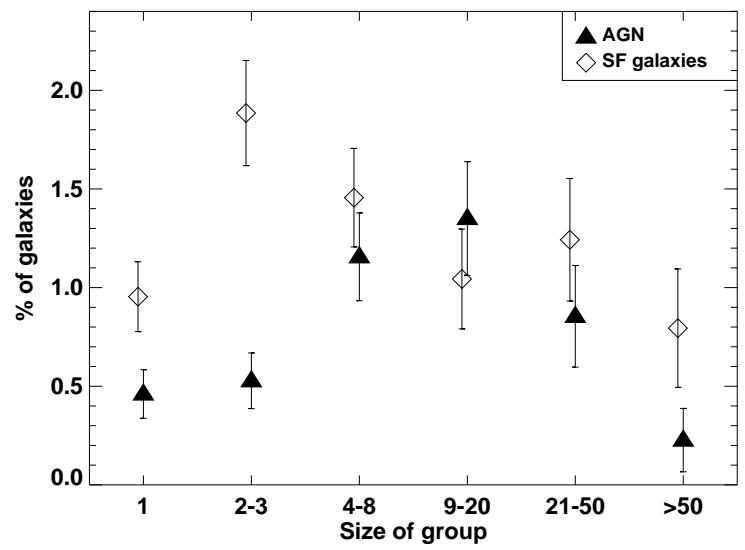

Figure 7. The fraction of galaxies which host radio-loud AGN or radioselected star forming galaxies as a function of the size of the group (as measured in $M_{B}<-19$ galaxies) in which the galaxy is located. Isolated galaxies are rarely luminous star forming galaxies, but beyond that star forming galaxies show a weak preference to be in the smallest groups possible. AGN are preferentially found in moderate groups and poor clusters.

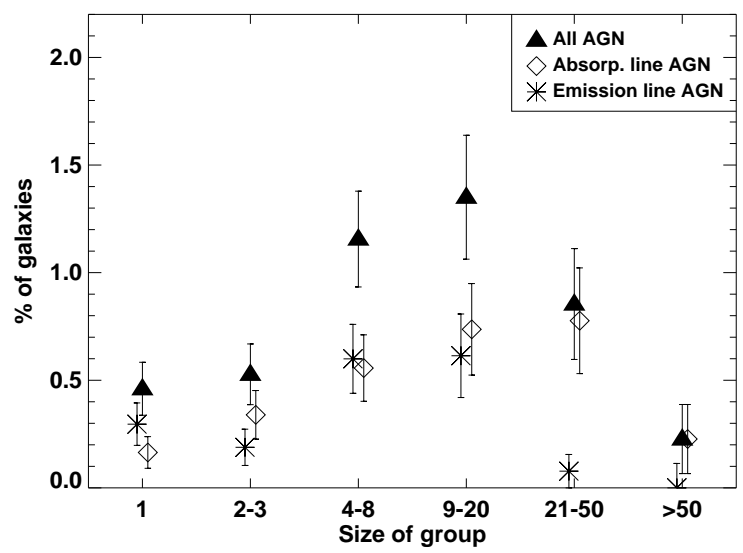

Figure 8. The fraction of galaxies which host radio-loud AGN as a function of the size of the group (as measured in $M_{B}<-19$ galaxies) in which the galaxy is located, split into subclasses of absorption-line AGN (Aa class of Sad02) and emission-line AGN (Ae and Aae classes). The emission-line AGN avoid even moderately rich clusters, while the absorption-line AGN lie preferentially in poor to moderate clusters, but are largely absent from rich clusters.

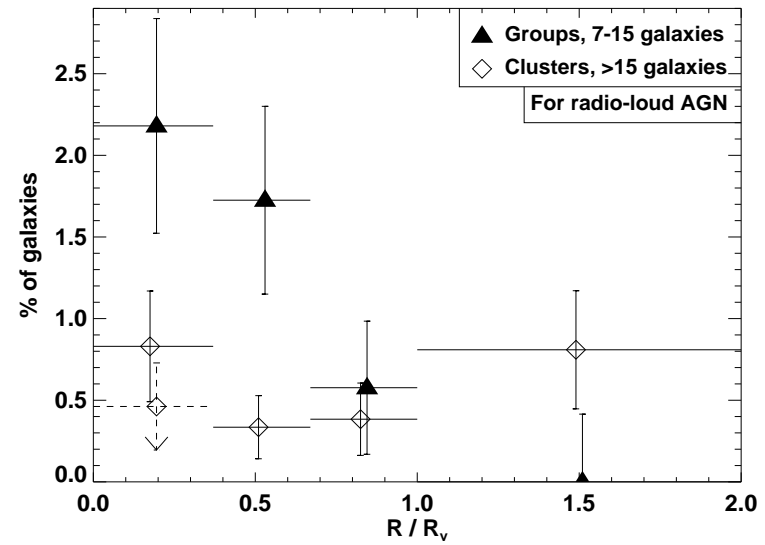

Figure 9. The radial distribution (in terms of projected radius of a galaxy from the group / cluster centre divided by the projected virial radius of that group / cluster) of the AGN fraction in both group (7-15 galaxies) and cluster ( $>15$ galaxies) environments. For the inner radial bin of the cluster AGN fraction, the lower data point represents the value after correcting for obvious brightest cluster galaxies. AGN are preferentially found in the inner regions of galaxy groups, but in clusters they are found at all radii, perhaps even preferentially at larger radii.

tion was also seen at the lowest surface densities. The decreased fraction of radio-loud AGN in the richest environments seen in the group membership analysis is, however, much more pronounced than anything in the galaxy surface densities. This apparent inconsistency arises because these two analyses sample environments on very different scales, which are not necessarily highly correlated: for example, a compact group of 10 galaxies can have a higher galaxy surface density than even the central regions of a diffuse cluster of 50 galaxies, while galaxies in a single rich clusters can span a wide range of local environments from very high galaxy surface densities at the cluster centre to much lower on the outskirts.

This latter point can be investigated by examining the variation of the AGN fraction with position in a group or cluster. Figure 9 shows that in galaxy groups the AGN are predominantly found in the central regions, whilst for clusters they are found across a wide range of radii. It is already known that brightest cluster galaxies often host AGN activity, and after correcting for these AGN (this correction may be even larger than that indicated on the figure, as only obvious brightest cluster galaxies were removed) there may even be a weak preference for AGN to lie at large radii if account is taken that some of the AGN at small projected radii may be at larger radii in a 3-dimensional analysis. 
It is interesting to compare the variations of AGN fraction in Figures 1 and 7: the large-scale environment (group, cluster, etc) of a galaxy seems to be more important than the small-scale environment (local galaxy surface density) in determining whether a galaxy will undergo AGN activity. This result is in contrast to that of the star formation properties of galaxies, where correlations are seen with both scales of environment but the stronger correlation is with the local galaxy density.

\section{DISCUSSION}

\subsection{Star forming galaxies}

For radio-selected star forming galaxies a strong correlation is seen between the fraction of galaxies which are undergoing star formation and the density of the local environment, in the sense that star formation is suppressed in higher density environments. This result is equivalent to that which has been found many times before in optical studies (e.g. Dressler et al. 1985; Balogh et al. 1998; Hashimoto et al. 1998; Carter et al. 2001; Lew02; Martínez et al. 2002; Gom03; MS03). The fraction of star forming galaxies is also found to be dependent upon the large-scale environment (group / cluster etc), in a similar way.

Lew02 found evidence for a density threshold at a projected galaxy surface density of $\sim 1$ galaxy per square Mpc, below which star formation is essentially independent of environment; Gom03 found a similar critical density threshold, although in this case the break is less clear and some residual environmental dependence remains at lower galaxy densities. No such threshold is seen in the current observations. In part this may be related to the fact that the radio selection only picks out the most rapidly star forming galaxies: Hashimoto et al. (1998) suggested that galaxies with high levels of star formation were very sensitive to local environment even outside of clusters, whilst those with lower star formation levels were less sensitive. However, this is unlikely to be the only effect since MS03 also found that the correlation of star formation activity with environment held across the full galaxy density range of their sample (as was also found in previous studies, e.g. Hashimoto et al. 1998). MS03 proposed three possible reasons to explain the difference between their results and those of Lew02 and Gom03. Firstly, MS03 restrict their analysis to field galaxies and so can convert redshifts into distances and use a volumetric local density estimate, as compared with the projected surface densities used by Lew02 and Gom03. Secondly, MS03 sample a magnitude further down the luminosity function than Lew02, and nearly two magnitudes fainter than Gom03; their sample will therefore include larger fractions of low luminosity star forming galaxies. Thirdly, the Lew02 results are based upon regions around clusters of galaxies, with the lowest densities corresponding to the cluster outskirts, and thus may give biased results with respect to a true field sample.

The results presented here provide some insight as to which of these factors is truly responsible. The magnitude limit of the current sample is equivalent to that of Lew02 and, like both Lew02 and Gom03, a projected surface density is used to estimate the local environment of the galaxies, yet no evidence for a critical density threshold is found. This suggests that neither of these two effects is responsible. More likely, the density threshold in the Lew02 studies arises as a result of restricting the analysis to only cluster environments. In particular, these authors select companion galaxies using a cut in velocity of three times the velocity dispersion of the clusters, as opposed to the fixed velocity width adopted here. It is not clear whether this technique is really appropriate in the 'field' environments at large cluster radii; the very wide velocity bins that result may contain large numbers of unrelated galaxies, and therefore introduce significant scatter into the surface density measurements in these rarefied environments, washing out any intrinsic correlation.

The results from the radio-selected star-forming galaxy sample support the idea that local environment plays a very important role in determining the star formation activity of a galaxy. This effect is still observed at very low galaxy densities, which suggests that ubiquitous processes such as gas stripping through tidal interactions and galaxy harassment are more important than ram pressure and evaporative stripping of gas by a hot intergalactic medium, which only function in cluster environments.

It is finally worth noting that the population of radio-selected dusty star-forming galaxies, found in the central regions of clusters by Miller \& Owen (2002), is clearly not a dominant contributant since the distribution of star forming galaxies selected on their radio emission (unaffected by dust) is comparable to that of opticallyselected samples.

\subsection{AGN Environments}

The fraction of galaxies hosting radio-loud AGN is seen to show little dependence upon the local galaxy surface density except in the very poorest environments. On the other hand, AGN show a strong preference to be found in galaxy groups or poor clusters, and tend to avoid both isolated environments and rich clusters. This latter result spectroscopically confirms the indications of previous imaging studies of the most powerful nearby radio sources (e.g. Prestage \& Peacock 1988; Hill \& Lilly 1991), and is also in line with the small-scale spectroscopic survey of Miller et al. (2002)

The AGN fraction appears to be much more strongly dependent upon the large-scale environment of a galaxy (group/cluster etc) than upon the small-scale environment evaluated through the local projected surface density of galaxies. This result has important consequences for understanding AGN activity. Radio-loud AGN do not simply trace the distribution of elliptical galaxies, and therefore the presence of a supermassive black hole, although essential, is not the principle driver of AGN activity. Nor is the general availability of cold gas the key factor, since the dependence of the AGN fraction on environment is radically different from that of the star forming population. There must be an alternative mechanism which in some way controls the triggering of the AGN activity. The environmental dependence of the AGN fraction strongly supports the argument that galaxy interactions or mergers provide this mechanism (e.g. Heckman et al. 1986): due to the lower velocity dispersions of galaxy groups, interactions and especially mergers are far more common in these environments than in the rich virialised environments of cluster cores. The observation that the AGN fraction is highest in the central regions of groups (where the galaxy density is highest) or the outer regions of clusters (perhaps infalling galaxies, or galaxy groups, more likely to undergo interactions) provides further support for this model.

One fundamental issue is whether radio-loud AGN are representative of all $\mathrm{AGN}$, in which case the results derived in this paper would be applicable to all AGN samples, or whether the radio selection produces some biased subsample. Until it is fully understood what causes an AGN to become radio-loud, this question cannot be definitively answered. However, studies of the most powerful radio-loud quasars have found no significant differences between either the host galaxies or the environments of these ob- 
jects and those of radio-quiet quasars selected to have the same optical luminosities (e.g. McLure et al. 1999; Nolan et al. 2001; McLure \& Dunlop 2001), except that radio-loud quasars are limited to the absolute upper end of the black hole mass function $\left(M_{\mathrm{bh}}>10^{9} M_{\odot}\right)$, whilst the radio quiet quasars extend to slightly lower values $\left(M_{\mathrm{bh}}>5 \times 10^{8} M_{\odot}\right.$; e.g. Dunlop et al. 2003). This suggests that, at least if analysis is restricted to the most powerful AGN, the radio-loud AGN are likely to be a reasonably unbiased subsample. At lower optical and radio luminosities the situation is less clear.

Given that the AGN fraction seems to depend more strongly on the large-scale than the local environment of their host galaxy, it will be interesting to investigate the dependence upon still larger scale. A visual comparison of the AGN and galaxy group/cluster distributions suggests that the AGN trace the large-scale structure of the groups and clusters, whilst avoiding the centres of the richest clusters; the very patchy redshift coverage of the 2dFGRS at the time that the Sad02 radio sample was constructed makes a quantitative analysis infeasible, however. Qualitatively, it is interesting to note there are already 9 radio-loud AGN associated with the largest supercluster structure in the 2dFGRS (at RA 13h, Dec $2^{\circ}, z \sim 0.084$, spanning a physical size of about $100 \mathrm{Mpc}$ eastwest), and the very incomplete redshift coverage for this supercluster when the Sad02 analysis took place suggests that this number will increase significantly. This is reminiscent of the recent results of Brand et al. (2003): these authors used the three-dimensional distribution of NVSS radio sources, as determined through a spectroscopic follow-up programme in dedicated sky regions, to discover at least one, and possibly two, $100 \mathrm{Mpc}-$ scale superstructures at $z \sim 0.3$ through radio source overdensities. These results suggest that studies of overdensities of radio sources in deep surveys may prove to be a powerful tool for discovering high redshift superclusters. In line with the argument above that radio-loud AGN are not a highly biased sample of AGN, it is not only radio sources which could be used for this: optical AGN overdensities may also trace these large structures (e.g. Williger et al. 2002; Haines et al. 2003), and especially the peripheral regions of these (e.g. Söchting et al. 2002).

\subsection{Absorption versus emission line AGN}

One of the most striking results to come out of the 2dFGRS analyses is the difference between the properties of the emission and absorption line AGN. Despite having comparable host galaxy properties and covering a similar range of radio luminosities, the environments of these two AGN classes differ dramatically. In low density environments there is a roughly equal split between absorption and emission line AGN, but in the densest environments essentially none of the AGN show strong line emission. This is reminiscent of the result of X-ray studies of nearby clusters, which found that the majority of the cluster X-ray AGN were associated with passive elliptical galaxies (Martini et al. 2002)

The difference between emission and absorption-line AGN may be related to that between 'normal, strong emission line' radio galaxies and the class of 'weak emission line' or 'low excitation' radio galaxies found within high radio power samples (Hine \& Longair 1979; Laing et al. 1994). In recent years it has been argued that low-excitation radio galaxies do not partake in the orientation-based unified schemes for radio sources (e.g. Barthel 1989), in which their stronger emission line counterparts are believed to be drawn from the same parent population as radio-loud quasars, but oriented such that direct quasar light is obscured by a torus of dusty material partially surrounding the nucleus. Harvanek et al. (2001) recently used galaxy number count analyses to show that radio galaxies at $z \sim 0.3$ typically live in richer environments than radio-loud quasars, and suggested that this might pose a problem for unified schemes. Hardcastle (2003) subsequently showed that this was not the case: if the low excitation radio galaxies are removed from the analysis, then there is no significant difference between the environments of strong emission line radio galaxies and those of the radio loud quasars. The entire difference in the Harvanek et al. (2001) study was driven by the low excitation radio galaxies, essentially all of which are found to reside in much richer environments than those of the strong emission line radio galaxies and quasars. This result exactly mirrors that derived in this paper, and suggests that these low excitation radio sources are simply higher radio power examples of the radio sources classified here as absorption-line AGN; the correlation between radio power and environmental richness found in this paper naturally explains why these high radio power low excitation radio sources typically live in very rich environments.

A key question is what drives this difference between the emission and absorption line AGN? Are these fundamentally different types of AGN, or does the surrounding environment influence the host galaxy to such an extent that different AGN properties are seen? Although fundamental differences in the AGN properties cannot be ruled out, the similarity of the host galaxies of the two different classes makes this hypothesis difficult to explain, whilst the alternative might be plausible through one or both of two different mechanisms.

Rawlings \& Saunders (1991) showed that for the strong emission line radio sources, there is a tight correlation between the jet power of the radio source and the emission line luminosity. Barthel \& Arnaud (1996) showed that radio sources in clusters have significantly more luminous radio emission than would be expected from their far-infrared luminosities, and argued that this was because the confining effect of the dense intracluster medium reduces adiabatic expansion losses in the radio lobes and therefore boosts the radio synchrotron emission (see Barthel \& Arnaud 1996, and references therein). The combination of these two effects suggests that some of the high radio power absorption line radio sources, in the denser environments, may be intrinsically lower jet-power AGN (with consequently lower expected emission line luminosities) whose location in a rich cluster has led to boosting of their radio emission giving rise to their comparatively high radio luminosities.

At first sight, this idea of boosted radio luminosities in cluster environments appears to be in contrast to indications that there is little difference between the radio luminosity functions inside and outside of clusters (e.g. Ledlow \& Owen 1996, and references therein). However, it is important to realise that the radio luminosity function arises through a complicated combination of the distribution of galaxy masses (since more luminous galaxies are more likely to host radio sources; e.g. Sadler et al. 1989), the triggering rate of radio sources, and the ultimate luminosities of those radio sources. A comparable radio luminosity function in clusters to that in the field may be obtained if fewer radio sources of a given intrinsic power are triggered in clusters (as suggested by the results of this paper), but each such radio source has higher radio luminosity. Clearly this issue of radio luminosity boosting is one which requires more detailed study.

An alternative explanation is that the absorption-line radio galaxies show little line emission, not because their AGN power is low, but because there is little gas in their surroundings capable of producing line emission. As has already been discussed for the 
analysis of star formation activity, a number of different physical mechanisms act to remove cool gas from galaxies in the vicinity of clusters, including tidal interactions, galaxy harassment, ram pressure stripping and evaporative stripping of gas by the hot intergalactic medium. Radio galaxies in cluster environments are therefore likely to be gas poor; there must be some cool gas supply available in order to fuel the central AGN, but this does not need to extend over the entire galaxy. If there is little cool gas in the galaxy, it follows directly that the covering fraction of these gas clouds will be low, and only a tiny proportion of the ionising photons emitted by the AGN will be intercepted, leading to very low emission line luminosities even for a relatively powerful AGN.

It seems probable that both of these mechanisms are important at some level. The first is able to explain the correlation between radio power and environment, whilst the second can explain why essentially all AGN in clusters show little or no line emission: boosting of the radio power alone cannot explain why X-ray sources in clusters also show a lack of line emission (cf. Martini et al. 2002). As an aside, it is worth noting that at high redshifts, powerful radio sources (with strong emission lines) are often found to lie in cluster environments, indicating that the separation between absorption and emission line AGN in rich environments breaks down at earlier cosmic epochs. This is likely to be because of the much greater availability of gas at these earlier epochs, even in young cluster environments.

Finally, this striking change in the ratio of absorption to emission line AGN in rich environments has important consequences for optically-based studies of AGN environments. In these studies AGN are selected on their emission-line properties, and so if this result for radio-loud AGN also holds for radio-quiet AGN, simple examination of the optical spectra will miss essentially all of the AGN in rich environments. Detailed modelling of the stellar populations of the galaxies, in order to recover weak emission lines within deep stellar absorption features, can help significantly here (Kauffmann et al, private communication), recovering weak emission line AGN. However, if the emission lines are significantly weaker or absent due to the lack of gas in cluster galaxies, then optical selection will miss some or many cluster AGN. This would distort any environmental analyses of optically-selected AGN, and may also introduce significant biases into other studies of AGN selected by these means.

\section{CONCLUSIONS}

The results of this paper can be summarised as follows:

- The proportion of radio-selected star forming galaxies decreases strongly with increasing local galaxy surface density, in the same manner as found in optical studies of star forming galaxies. This correlation extends over the full range of galaxy surface densities, with no evidence for a lower density threshold.

- Radio-loud AGN activity shows little dependence on local galaxy surface density, except at the very lowest surface densities where little AGN activity is found. The larger scale environment is more important in determining AGN activity: AGN are preferentially found in moderate groups and poor clusters.

- The AGN activity traces neither the distribution of galaxy bulges nor the availability of cold gas in galaxies, meaning that an external influence is required to trigger the activity. The higher AGN fraction in environments where conditions are optimised for galaxy interactions and mergers indicates that these are likely to be an important mechanism.
- Where AGN are found in poor or moderate richness clusters they are almost invariably absorption-line AGN, and have relatively high radio luminosities. This likely reflects the lack of cool gas typically available for ionisation in cluster environments, and suggests that the radio luminosity of these sources may have been boosted by their dense surrounding environment.

- The substantial drop in the ratio of emission-line to absorption-line AGN in dense environments implies that, at very least, considerable care must be taken in selecting samples of AGN from their optical emission-line properties. Potentially these samples could miss a large fraction of cluster AGN, in which case results from AGN environmental studies based upon opticallyselected AGN samples would be unreliable.

An investigation of the environments of radio-selected AGN over a much larger area, such as that which will ultimately be possible using the SDSS, will permit these environmental variations to be studied to a much greater degree, using a radio sample of sufficient size for more detailed statistical investigation of AGN subsamples. In addition, such studies would enable a detailed comparison between optically and radio selected AGN samples; this is the key to understanding the potential biases of each method.

It is also important to investigate more powerful AGN than those studied here: the most powerful radio source in the current study has a $1.4 \mathrm{GHz}$ radio luminosity of $1.4 \times 10^{25} \mathrm{~W} \mathrm{~Hz}^{-1}$, while the most powerful nearby sources have radio luminosities of $10^{26-27} \mathrm{~W} \mathrm{~Hz}^{-1}$, much more comparable to those found at higher redshifts. Studies of these sources would permit an investigation of the cosmic evolution of AGN environments, but such powerful AGN are very rare in the nearby Universe, and a dedicated redshift survey of their environments will be required to achieve this.

\section{ACKNOWLEDGEMENTS}

The author would like to thank the Royal Society for financial support through its University Research Fellowship scheme, Guinevere Kauffmann for detailed discussions of optical versus radio selection of AGN, and Ignas Snellen for a number of useful discussions. The author is also grateful to the referee for prompt and helpful comments on the original version of the manuscript. This research has made use of the $2 \mathrm{dFGRS}$, the data for which has been reduced and released by the 2dFGRS team, to whom the author is grateful. A modified version of the 2dFGRS mask software of Peder Norberg and Shaun Cole was also used. The research makes use of the SDSS Archive, funding for the creation and distribution of which was provided by the Alfred P. Sloan Foundation, the Participating Institutions, the National Aeronautics and Space Administration, the National Science Foundation, the U.S. Department of Energy, the Japanese Monbukagakusho, and the Max Planck Society.

\section{REFERENCES}

Abazajian K. et al. 2003, AJ, 126, 2081

Balogh M. L., Schade D., Morris S. L., Yee H. K. C., Carlberg R. G., Ellingson E., 1998, ApJ, 504, 75

Barthel P. D., 1989, ApJ, 336, 606

Barthel P. D., Arnaud K. A., 1996, MNRAS, 283, L45

Beers T. C., Flynn K., Gebhardt K., 1990, AJ, 100, 32

Biviano A., Katgert P., Mazure A., Moles M., den Hartog R., Perea J., Focardi P., 1997, A\&A, 321, 84

Brand K., Rawlings S., Hill G. J., Lacy M., Mitchell E., Tufts J., 2003, MNRAS, 306, 283 
Carilli C. L., 2001, in Tacconi L., Lutz D., eds, Starburst Galaxies: Near and Far. Springer-Verlag, Heidelberg, p. 309

Carter B. J., Fabricant D. G., Geller M., Kurtz M. J., McLean B., 2001, ApJ, 559,606

Colless M. et al. 2001, MNRAS, 328, 1039

Condon J. J., 1992, ARA\&A, 30, 575

Condon J. J., Cotton W. D., Greisen E. W., Yin Q. F., Perley R. A., Taylor G. B., Broderick J. J., 1998, AJ, 115, 1693

Dressler A., 1980, ApJ, 236, 351

Dressler A., Thompson I. B., Shectman S. A., 1985, ApJ, 288, 481

Dunlop J. S., 1998, in Bremer M. N., Jackson N., Pérez-Fournon I., eds, Observational Cosmology with the New Radio Surveys. Kluwer Academic Publishers, Dordrecht

Gómez P. et al. 2003, ApJ, 584, 210 [Gom03]

Giraldi M., Biviano A., Giuricin G., Mardirossian F., Mezzetti M., 1993, ApJ, 404, 38

Giraldi M., Giuricin G., 2000, ApJ, 540, 45

Gunn J. E., Gott J. R., 1972, ApJ, 176, 1

Haines C. P., Campusano L. E., Clowes R. G., 2003, A\&A, in press

Hardcastle M. J., 2003, A\&A, in press

Harvanek M., Ellingson E., Stocke J. T., Rhee G., 2001, AJ, 122, 2874

Hashimoto Y., Oemler Jr. A., Lin H., Tucker D. L., 1998, ApJ, 499, 589

Heckman T. M., Bothun G. D., Balick B., Smith E. P., 1984, AJ, 89, 958

Heckman T. M., Smith E. P., Baum S. A., van Breugel W. J. M., Miley G. K., Illingworth G. D., Bothun G. D., Balick B., 1986, ApJ, 311, 526

Hill G. J., Lilly S. J., 1991, ApJ, 367, 1

Hine R. G., Longair M. S., 1979, MNRAS, 188, 111

Ho L. C., Filippenko A. V., Sargent W. L. W., 1997, ApJ, 487, 579

Hooper E. J., Impey C. D., Foltz C. B., Hewitt P. C., 1995, ApJ, 445, 62

Huchra J. P., Geller M. J., 1982, ApJ, 257, 423

Hutchings J. B., Campbell B., 1983, Nat, 303, 584

Kauffmann G. et al. 2003, MNRAS, submitted; astro-ph/0304239

Kormendy J., Gebhardt K., 2001, p. 363

Laing R. A., Jenkins C. R., Wall J. V., Unger S. W., 1994, in Bicknell G. V., Dopita M. A., Quinn P. J., eds, The first Stromlo symposium: Physics of active galaxies. Cambridge University Press, Cambridge, p. 201

Ledlow M. J., Owen F. N., 1996, AJ, 112, 9

Lewis I et al. 2002, MNRAS, 334, 673 [Lew02]

Machalski J., Godlowski W., 2000, A\&A, 360, 463

Madau P., Pozzetti L., Dickinson M., 1998, ApJ, 498, 106

Madgwick D. S. et al. 2002, MNRAS, 333, 133

Martínez H. J., Zandivarez A., Domínguez M., Merchán M. E., Lambas D. G., 2002, MNRAS, 333, L31

Martini P., Kelson D. D., Mulchaey J. S., Trager S. C., 2002, ApJ, 576, 109

Mateus Jr. A., Sodré Jr. L., 2003, MNRAS, submitted; astro-ph/0307349 [MSO3]

McLure R. J., Dunlop J. S., 2001, MNRAS, 321, 515

McLure R. J., Kukula M. J., Dunlop J. S., Baum S. A., O’Dea C. P., Hughes D. H., 1999, MNRAS, 308, 377

Merchán M., Zandivarez A., 2002, MNRAS, 335, 216

Miller C. J., Nichol R. C., Goméz P. L., Hopkins A. M., Bernardi M., 2003, ApJ in press; astro-ph-0307124

Miller N. A., Ledlow M. J., Owen F. N., Hill J. M., 2002, AJ, 123, 3018

Miller N. A., Owen F. N., 2002, AJ, 124, 2453

Nolan L. A., Dunlop J. S., Kukula M. J., Hughes D. H., Boroson T., Jimenez R., 2001, MNRAS, 323, 308

Nolthenius R., White S. D. M., 1987, MNRAS, 235, 505

Norberg P. et al. 2002, MNRAS, 336, 907

Norberg P. et al. 2003, Ap. \& Sp. Sci., 285, 205

Prestage R. M., Peacock J. A., 1988, MNRAS, 230, 131

Ramella M., Pisani A., Geller M. J., 1997, ApJ, 113, 483

Rawlings S., Saunders R., 1991, Nat, 349, 138

Sadler E. M., Jenkins C. R., Kotanyi C. G., 1989, MNRAS, 240, 591

Sadler E. M. et al. 2002, MNRAS, 329, 227 [Sad02]

Söchting I. K., Clowes R. G., Campusano L. E., 2002, MNRAS, 331, 569

Stoughton C. et al. 2002, AJ, 123, 485

Strateva I. et al. 2001, AJ, 122, 1861

Wainer H., Thissen D., 1976, Psychometrika, 41, 9
Williger G. M., Campusano L. E., Clowes R. G., Graham M. J., 2002, ApJ, 578,708

York D. G. et al. 2000, AJ, 120, 1579 